\title{
Sensitive cardiac troponin I predicts poor outcomes in pulmonary arterial hypertension
}

\author{
G.A. Heresi*, W.H.W. Tang" ${ }^{\#}$, M. Aytekin`, J. Hammel*, S.L. Hazen" and R.A. Dweik* ${ }^{*}$
}

ABSTRACT: Circulating cardiac troponins are markers of myocardial injury. We sought to determine whether cardiac troponin I (cTnl), measured by a sensitive assay, is associated with disease severity and prognosis in pulmonary arterial hypertension (PAH).

cTnl was measured in 68 patients with PAH diagnostic category 1 in a research-based sensitive immunoanalyser with a lower limit of detection of $0.008 \mathrm{ng} \cdot \mathrm{mL}^{-1}$. The associations between $\mathrm{cTnl}$ and PAH severity and clinical outcomes were assessed using Chi-squared and Wilcoxon rank sum tests, Kaplan-Meier analysis and Cox regression models.

cTnl was detected in $25 \%$ of patients. Patients with detectable cTnl had more advanced functional class symptoms, a shorter 6-min walk distance, more pericardial effusions, larger right atrial area, and higher B-type natriuretic peptide and C-reactive protein levels. 36-month transplant-free survival was $44 \%$ in patients with detectable cTnl versus $85 \%$ in those with undetectable cTnl. cTnl was associated with a 4.7-fold increased risk of death related to right ventricular failure or transplant (hazard ratio $4.74,95 \% \mathrm{Cl} 1.89-11.89 ; \mathrm{p}<0.001$ ), even when adjusted individually for known parameters of PAH severity.

Elevated plasma cTnl, even at subclinically detectable levels, is associated with more severe disease and worse outcomes in patients with PAH.

KEYWORDS: Biomarkers, cardiac troponin, outcomes, prognosis, pulmonary arterial hypertension

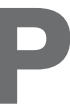

ulmonary arterial hypertension $(\mathrm{PAH})$ is characterised by luminal obliteration and remodelling of the pulmonary vasculature leading to progressive increments in pulmonary vascular resistance. This leads to overload of the right ventricle, right ventricular failure (RVF) and premature death [1]. Despite impressive advancements in the understanding of the pathobiology of $\mathrm{PAH}$ and the introduction of targeted medical therapies, the mortality associated with this condition remains substantial [2]. While guidelines suggest how to tailor different therapeutic approaches according to the severity of the disease [3], risk stratification is difficult and often relies on suboptimal tools such as the assessment of symptoms via the New York Heart Association (NYHA) functional classification. Noninvasive biomarkers of the disease offer tremendous potential to aid difficult therapeutic decisions [4].

The detection of circulating cardiac troponins reflects myocardial necrosis and is diagnostic of acute myocardial infarction in the presence of ischaemic signs and symptoms [5]. In recent years, it has been recognised that elevated cardiac troponins may be detected in other conditions, including stable coronary artery disease [6], left ventricular failure [7], chronic kidney disease [8], sepsis [9] and mixed critical care populations [10]. Elevated cardiac troponins have also been noted in situations of right ventricular overload, both acute, as in acute pulmonary embolism [11], and chronic, as in pulmonary hypertension $[12,13]$. Mechanisms other that myocardial necrosis may be responsible for this "troponin leak" in these clinical scenarios, even though detectable cardiac troponin is specific for ongoing myocardial damage and generally associated with worse prognosis. In this study, we sought to determine if cardiac troponin I (cTnI), measured with a sensitive assay, is associated with severity and prognosis in diagnostic category 1 $\mathrm{PAH}$ patients treated with contemporary $\mathrm{PAH}-$ targeted therapy.

\section{AFFILIATIONS}

*Pulmonary and Critical Care Medicine, Respiratory Institute, ${ }^{*}$ Cardiovascular Medicine, Heart and Vascular Institute, and "Pathobiology, Lerner Research Institute, Cleveland Clinic, Cleveland, $\mathrm{OH}$, USA.

CORRESPONDENCE

R.A. Dweik

Pulmonary and Critical Care Medicine

Respiratory Institute Cleveland Clinic

9500 Euclid Avenue A90

Cleveland

$\mathrm{OH} 44195$

USA

E-mail: dweikr@ccf.org

Received:

April 192011

Accepted after revision:

Aug 102011

First published online:

Sept 012011 


\section{MATERIALS AND METHODS}

\section{Study population and clinical data collection}

Patients were recruited from the Cleveland Clinic Pulmonary Vascular Program (Cleveland, OH, USA). Eligible subjects included patients with PAH diagnostic category 1 according to the updated Dana Point clinical classification [14]. Acute coronary syndromes and advanced renal disease, defined as a serum creatinine $>2 \mathrm{mg} \cdot \mathrm{dL}^{-1}$, were further exclusion criteria. Nonconsecutive patients were enrolled after their peripheral blood was collected for study purposes. Venous blood was obtained at the time of right heart catheterisation in 17 incident (newly diagnosed) patients, or during a routine clinical visit where 6-min walk distance (6MWD), NYHA functional class, Doppler echocardiography and brain natriuretic peptide (BNP) measurements were performed in the remaining 51 patients with prevalent disease. Blood was processed and samples were stored in a biobank until biomarker measurements were performed. Time to clinical outcomes was determined from the time of blood sampling. The clinical outcome of interest was transplant-free survival. Events for this outcome were either lung transplantation or death by RVF. Patients who were alive at the end of follow-up and those who died of causes other than RVF were censored. The cause of death was determined by G.A. Heresi by careful review of the medical records and, when needed, discussions with the treating clinician. Clinical events and data were recorded in a prospective fashion in 48 patients and retrospectively in the remaining 20. 6MWD, NYHA functional class, echocardiographic data, and serum creatinine and BNP levels were measured on the same day that study blood sampling took place. Right heart catheterisation data were obtained as close to study blood sampling as possible. The median time between diagnostic right heart catheterisation and blood sampling for study purposes was 240 days. Other data collected included demographic information, traditional cardiovascular risk factors (diabetes mellitus, systemic arterial hypertension and smoking status), coronary artery disease, serum creatinine levels and the use of PAH-targeted therapies. Healthy volunteers were used as controls. This study was approved by the Cleveland Clinic Institutional Review Board (Cleveland Clinic, Cleveland, OH, USA). All study subjects gave written informed consent.

\section{Laboratory measurements}

Plasma levels of cTnI were measured using the STAT Troponin I assay (Abbott Laboratories, Inc., Abbott Park, IL, USA) in a research-based immunoanalyser that provides a three-decimal point readout from venous blood samples collected in EDTA tubes. Levels $\geqslant 0.03 \mathrm{ng} \cdot \mathrm{mL}^{-1}$ are considered to be diagnostic of myocardial infarction in the right clinical context. We have previously shown that this assay provides highly sensitive analytical measurement of cTnI with a limit of detection of $0.008 \mathrm{ng} \cdot \mathrm{mL}^{-1}$ [15]. However, the assay detected cTnI levels between 0.001 and $0.008 \mathrm{ng} \cdot \mathrm{mL}^{-1}$ in 1,291 subjects out of a total of 3,828 stable patients undergoing an elective cardiac evaluation. Importantly, cTnI levels between 0.001 and $0.008 \mathrm{ng} \cdot \mathrm{mL}^{-1}$ were associated with an increased risk of death, myocardial infarction or stroke compared with levels $<0.001 \mathrm{ng} \cdot \mathrm{mL}^{-1}$ [15]. This assay has also been shown to be comparable with other high-sensitivity assays [16]. Thus, for the purpose of this study, we defined detectable $\mathrm{cTnI}$ as any level $>0.001 \mathrm{ng} \cdot \mathrm{mL}^{-1}$. In the
PAH cohort, we also measured high-sensitivity C-reactive protein (CRP) and high-density lipoprotein cholesterol (HDL-C) using the Abbott ARCHITECT platform. We also measured cardiac troponin T (cTnT) with the Elecsys Troponin $\mathrm{T}$ assay (Roche Diagnostics, Indianapolis, IN, USA) in 62 out of the 68 patients. This assay provides a two-decimal readout with a limit of detection of $0.01 \mathrm{ng} \cdot \mathrm{mL}^{-1}$

\section{Statistical analysis}

Demographic, clinical and laboratory data were summarised as frequency $(\%)$ and mean $\pm \mathrm{SD}$. cTnI levels were compared between PAH patients and controls by means of Wilcoxon rank sum and Chi-squared tests. Patient groups defined by cTnI status were compared with respect to categorical study variables using Chi-squared and Fisher's exact test, and with respect to quantitative and ordinal variables using Wilcoxon rank sum tests. Correlations between cTnI and variables of interest were estimated by the Spearman correlation coefficient.

The association between transplant-free survival and cTnI was assessed using log-rank tests, with Kaplan-Meier estimation used to describe the likelihood of the outcomes during the follow-up period. Patients who died of causes other than RVF were censored for this analysis. Cox regression models were used to provide estimates of hazard ratios and $95 \%$ confidence intervals for transplant-free survival with respect to their associations with cTnI. Cox regression was also used in a multivariable fashion to explore the covariate-adjusted associations between transplant-free survival and cTnI. We adjusted for selected covariates individually, as the sample size and number of events did not allow for building models with multiple covariates. Results for covariate adjusted associations were summarised using Wald p-values and covariate-adjusted hazard ratio estimates. Analyses were performed using $\mathrm{R}$ version 2.8.1 (R Development Core Team 2011; R Foundation for Statistical Computing, Vienna, Austria) [17].

\section{RESULTS}

\section{Study population and cTnI levels}

We studied $68 \mathrm{PAH}$ patients (aged mean \pm SD $47 \pm 13 \mathrm{yrs}, 91 \%$ female) and 29 controls (aged $35 \pm 6$ yrs, $75 \%$ female). PAH categories were as follows: idiopathic PAH $(n=41)$; heritable PAH (hereditary haemorrhagic telangiectasia; $n=1$ ); and associated PAH $(n=26 ; 20$ connective tissue disease, three congenital heart disease, two chronic haemolytic anaemia and one portopulmonary). At the time of study enrolment, 51 (75\%) patients were receiving PAH-targeted therapies: intravenous prostacyclin $(\mathrm{n}=15)$; phosphodiesterase inhibitor (PDE5i) $(n=7)$; endothelin receptor antagonist (ERA) $(n=4)$; i.v. prostacyclin plus ERA $(\mathrm{n}=13) ;$ i.v. prostacyclin plus PDE5i $(\mathrm{n}=3)$; ERA plus PDE5i $(\mathrm{n}=5)$; and i.v. prostacyclin plus ERA plus PDE5i $(n=4)$. The 17 patients naive to therapy at enrolment (25\% of the cohort) were eventually treated as follows: i.v. prostacyclin $(\mathrm{n}=2)$; PDE5i $(\mathrm{n}=4)$; i.v. prostacyclin plus PDE5i $(\mathrm{n}=4)$; ERA plus PDE5i $(\mathrm{n}=3)$; i.v. prostacyclin plus PDE5i $(n=2)$; and i.v. prostacyclin plus ERA plus PDE5i $(n=2)$. Table 1 details functional, echocardiographic and haemodynamic characteristics of the PAH cohort. No PAH patient had diabetes, 15 (22\%) had systemic hypertension and $27(40 \%)$ were either former or active smokers. Three $(4 \%)$ patients had coronary artery disease, none of whom had detectable cTnI. 


\begin{tabular}{|c|c|c|c|c|}
\hline \multirow[t]{2}{*}{ TABLE 1} & \multicolumn{3}{|c|}{$\begin{array}{l}\text { aracteristics of the pulmonary arterial } \\
\text { ertension cohort }\end{array}$} & \multirow[b]{2}{*}{ p-value ${ }^{\#}$} \\
\hline & $\begin{array}{c}\text { All } \\
\text { patients }\end{array}$ & $\begin{array}{c}\text { Undetectable } \\
\text { cTnl }\end{array}$ & $\begin{array}{c}\text { Detectable } \\
\text { cTnl }\end{array}$ & \\
\hline Subjects & 68 & 51 & 17 & \\
\hline Age yrs & $47 \pm 13$ & $46 \pm 12$ & $49 \pm 16$ & 0.42 \\
\hline Female & $62(91)$ & 47 (92) & $15(88)$ & 0.64 \\
\hline АРАH & $27(40)$ & $16(31)$ & $11(65)$ & 0.019 \\
\hline NYHA III-IV & $44(65)$ & $29(57)$ & $15(88)$ & 0.031 \\
\hline 6MWD m & $367 \pm 124$ & $389 \pm 119$ & $297 \pm 116$ & 0.012 \\
\hline Pericardial effusion & $22(33)$ & $12(24)$ & $10(59)$ & 0.011 \\
\hline $\mathrm{RA}$ area $\mathrm{cm}^{2}$ & $24 \pm 8$ & $22 \pm 7$ & $29 \pm 9$ & 0.011 \\
\hline Pra $\mathrm{mmHg}$ & $11 \pm 6$ & $10 \pm 6$ & $13 \pm 6$ & 0.06 \\
\hline $\bar{P}_{\text {pa }} \mathbf{m m H g}$ & $52 \pm 14$ & $51 \pm 12$ & $56 \pm 17$ & 0.35 \\
\hline $\mathrm{CI} L \cdot \mathrm{min}^{-1} \cdot \mathrm{m}^{-2}$ & $2.27 \pm 0.81$ & $2.33 \pm 0.83$ & $2.09 \pm 0.72$ & 0.36 \\
\hline PVR Wood units & $11 \pm 7$ & $10 \pm 6$ & $14 \pm 10$ & 0.24 \\
\hline BNP $\mathrm{pg} \cdot \mathrm{mL}^{-1}$ & $224 \pm 314$ & $155 \pm 280$ & $454 \pm 321$ & $<0.001$ \\
\hline CRP $\mathrm{mg} \cdot \mathrm{L}^{-1}$ & $12.7 \pm 20.9$ & $7.8 \pm 8.6$ & $27.5 \pm 35.8$ & 0.007 \\
\hline $\mathrm{HDL}-\mathrm{C} \mathbf{m g} \cdot \mathrm{dL}^{-1}$ & $35 \pm 11$ & $38 \pm 10$ & $27 \pm 11$ & 0.001 \\
\hline Creatinine $\mathrm{mg} \cdot \mathrm{dL}^{-1}$ & $0.90 \pm 0.26$ & $0.89 \pm 0.24$ & $0.94 \pm 0.32$ & 0.58 \\
\hline
\end{tabular}

Data are presented as $n$, mean \pm SD or $n(\%)$, unless otherwise indicated. $C T n l$ : cardiac troponin I; APAH: associated pulmonary arterial hypertension; NYHA: New York Heart Association functional class; 6MWD: 6-min walk distance; RA: right atrial; Pra: right atrial pressure; $\bar{P}$ pa: mean pulmonary artery pressure; $\mathrm{Cl}$ : cardiac index; PVR: pulmonary vascular resistance; BNP: brain natriuretic peptide; CRP: C-reactive protein; HDL-C: high-density lipoprotein cholesterol. \#: comparison between detectable and undetectable cTnl.

17 (25\%) PAH patients had detectable cTnI levels (>0.001 ng. $\left.\mathrm{mL}^{-1}\right)$, compared with one $(3.4 \%)$ control $(\mathrm{p}=0.011)$. Two $(2.9 \%)$ patients had cTnI levels between 0.001 and $0.008 \mathrm{ng}$. $\mathrm{mL}^{-1}$. Nine $(13.2 \%)$ patients had cTnI levels between 0.009 and $0.029 \mathrm{ng} \cdot \mathrm{mL}^{-1}$, as did the one control with detectable cTnI. Six $(8.8 \%)$ patients had levels $>0.030 \mathrm{ng} \cdot \mathrm{mL}^{-1}$, none of whom had coronary artery disease. In patients with detectable cTnI, mean \pm SD levels were $0.037 \pm 0.009 \mathrm{ng} \cdot \mathrm{mL}^{-1}$, with a range of $0.003-0.140 \mathrm{ng} \cdot \mathrm{mL}^{-1}$. The detection rate for $\mathrm{cTnT}$ was only four $(6.5 \%)$ out of $62(\mathrm{p}=0.006$ for the comparison with the $25 \%$ detection rate of cTnI). All patients with detectable cTnT also had a detectable cTnI. Conversely, 12 patients with detectable cTnI had undetectable cTnT.

\section{cTnI and PAH severity}

cTnI concentrations had significant positive correlations with NYHA functional class, right atrial (RA) area measured with echocardiography, and BNP and CRP levels (table 2). There was a borderline positive correlation with right atrial pressure (Pra) $(\mathrm{r}=0.24, \mathrm{p}=0.06)$. Negative correlations with 6MWD, mixed venous oxygen saturation and HDL-C levels $(r=-0.42)$ were observed (table 2).

Patients with detectable cTnI were more likely to have associated PAH, more advanced functional class symptoms and more pericardial effusions; they also had shorter 6MWD, higher Pra, larger RA area, higher BNP and CRP levels, and lower HDL-C concentrations (table 1). Both subgroups were similar in age, sex, cardiac frequency and systemic blood pressure, as well as in the proportion of subjects with systemic hypertension and tobacco use.

\section{Clinical outcomes}

$22(32.4 \%)$ patients died and two (2.9\%) underwent lung transplantation. Nine (53\%) out of 17 patients with detectable cTnI died, compared with 13 (25\%) out of 51 with undetectable $c \operatorname{TnI}(p=0.041)$. All of the nine deaths in patients with detectable cTnI were due to RVF. Among the 13 deaths with undetectable cTnI, three were secondary to severe infections (two pneumonias and one catheter-related bloodstream infection), one was related to pancreatic cancer and one could not be ascertained; the remaining eight deaths were secondary to RVF. There were no sudden deaths in either group. The proportion of transplant or death related to RVF in patients with undetectable cTnI was $17.6 \%$ (nine out of 51) compared with $58.8 \%$ (10 out of 17) in patients with detectable cTnI $(p=0.003) .15(26 \%)$ out of 58 patients with undetectable cTnT died of RVF at the end of follow-up, compared with three out of four patients $(75 \%)$ with detectable cTnT $(\mathrm{p}=0.07)$.

Detectable cTnI was associated with a 4.7-fold increased risk of death related to RVF or transplant: hazard ratio (HR) $4.74,95 \%$ CI 1.89-11.89 ( $\mathrm{p}<0.001)$ (fig. 1 and table 3$)$. In patients with detectable cTnI, 12-, 24- and 36-month transplant-free survival was $64.7,52.9$ and $44.1 \%$ respectively, compared with $96.1,90$ and $84.7 \%$ in patients with undetectable cTnI. Table 3 shows that age, type of PAH, NYHA functional class, 6MWD, pericardial effusion, Pra and BNP were associated with the risk of death by RVF or transplant. We also found prognostic values for HDL-C and creatinine, but not for CRP. In this cohort, there was no association between prevalent versus incident cases and different PAH-targeted therapies with clinical outcomes (table 3 ). The association between cTnI and transplant-free survival remained unchanged when adjusted individually for age, PAH subcategory (idiopathic versus associated), NYHA functional class, 6MWD, the presence of pericardial effusion, Pra, cardiac index, the use prostacyclin therapy, and HDL-C, CRP and creatinine concentrations (all $\mathrm{p}<0.05$ ) (table 4). Adjustment for the presence of systemic hypertension, coronary artery disease, statin therapy and body

\begin{tabular}{lccc}
\hline TABLE 2 & $\begin{array}{l}\text { Significant correlations between cardiac troponin } \\
\text { I and pulmonary arterial hypertension indices of } \\
\text { severity }\end{array}$ \\
Correlation & Variable & $\mathbf{r}$ & p-value \\
\hline \multirow{2}{*}{ Positive } & BNP & 0.45 & $<0.001$ \\
& NYHA class & 0.36 & 0.002 \\
& CRP & 0.34 & 0.004 \\
Negative & RA area & 0.36 & 0.010 \\
& HDL-C & -0.42 & $<0.001$ \\
& 6MWD & -0.29 & 0.020 \\
& Sv, $\mathrm{O}_{2}$ & -0.30 & 0.034 \\
\hline
\end{tabular}

BNP: brain natriuretic peptide; NYHA: New York Heart Association functional class; CRP: C-reactive protein; RA: right atrial; $\mathrm{HDL}-\mathrm{C}$ : high-density lipoprotein cholesterol; 6MWD: 6-min walk distance; $\mathrm{Sv}, \mathrm{O}_{2}$ : mixed venous oxygen saturation. 


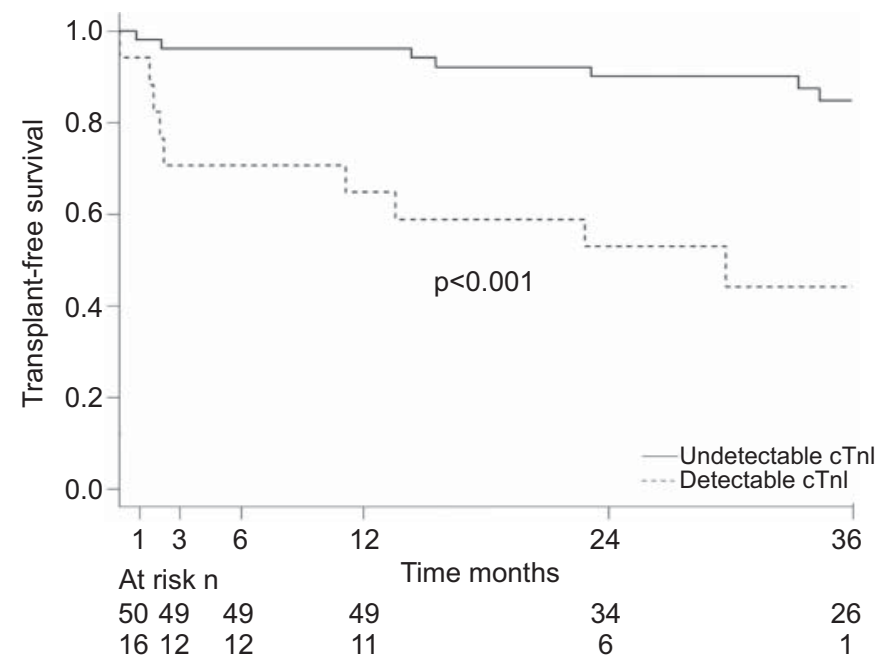

FIGURE 1. Transplant-free survival according to cardiac troponin I (cTnl) status. Patients with detectable $\mathrm{CTnl}$ had a 4.7-fold higher risk of right ventricularrelated death or lung transplantation compared to patients with undetectable cTnl.

mass index also did not affect this association. There was a significant interaction between cTnI and BNP $(p=0.006)$. Patients with both low BNP levels and undetectable cTnI had longer transplant-free survival, while those with both detectable cTnI and supra-median BNP had the worst outcomes, but

\begin{tabular}{|c|c|c|c|}
\hline \multicolumn{2}{|l|}{ Variable } & Univariable HR $(95 \% \mathrm{Cl})$ & p-value \\
\hline \multicolumn{2}{|c|}{ Detectable cTnI } & $4.74(1.89-11.89)$ & $<0.001$ \\
\hline \multicolumn{2}{|l|}{ Age $^{\#}$ yrs } & $1.70(1.01-2.87)$ & 0.048 \\
\hline \multicolumn{2}{|c|}{ IPAH versus APAH } & $0.39(0.15-0.99)$ & 0.048 \\
\hline \multicolumn{2}{|l|}{ NYHA class } & $3.55(1.03-12.2)$ & 0.045 \\
\hline \multicolumn{2}{|l|}{$6 \mathrm{MWD}^{\#} \mathrm{~m}$} & $0.60(0.37-0.97)$ & 0.038 \\
\hline \multicolumn{2}{|c|}{ Pericardial effusion } & $3.21(1.28-8.06)$ & 0.013 \\
\hline \multicolumn{2}{|l|}{$\operatorname{Pra}^{\#} \mathrm{mmHg}$} & $2.17(1.30-3.61)$ & 0.003 \\
\hline \multicolumn{2}{|c|}{$\mathrm{Cl}^{\#} \mathrm{~L} \cdot \mathrm{min}^{-1} \cdot \mathrm{m}^{-2}$} & $1.00(0.64-1.56)$ & 0.99 \\
\hline \multicolumn{2}{|c|}{$\mathrm{CRP}^{\#} \mathrm{mg} \cdot \mathrm{L}^{-1}$} & $1.17(0.83-1.63)$ & 0.37 \\
\hline \multicolumn{2}{|c|}{ HDL-C ${ }^{\#} \mathrm{mg} \cdot \mathrm{dL}^{-1}$} & $0.45(0.28-0.71)$ & 0.001 \\
\hline \multicolumn{2}{|c|}{ Creatinine $^{\#} \mathrm{mg} \cdot \mathrm{dL}^{-1}$} & $1.50(1.05-2.14)$ & 0.027 \\
\hline \multicolumn{2}{|l|}{ BNP $^{\#}$} & $2.01(1.39-2.90)$ & $<0.001$ \\
\hline \multicolumn{2}{|c|}{ Prevalent versus incident disease } & $1.39(0.45-4.22)$ & 0.57 \\
\hline \multicolumn{2}{|l|}{ Prostacyclin } & $1.28(0.49-3.30)$ & 0.61 \\
\hline \multicolumn{2}{|l|}{ ERA } & $1.18(0.48-2.91)$ & 0.72 \\
\hline \multicolumn{2}{|l|}{ PDE5i } & $0.63(0.25-1.59)$ & 0.32 \\
\hline \multicolumn{2}{|c|}{ PAH medications ${ }^{\#}$} & $0.99(0.62-1.57)$ & 0.96 \\
\hline
\end{tabular}

HR: hazard ratio; cTnl: cardiac troponin I; IPAH: idiopathic pulmonary arterial hypertension; APAH: associated pulmonary arterial hypertension; NYHA: New York Heart Association functional class; 6MWD: 6-min walk distance; Pra: right atrial pressure; $\mathrm{Cl}$ : cardiac index; CRP: C-reactive protein; HDL-C: high-density lipoprotein cholesterol; BNP: B-type natriuretic peptide; ERA: endothelin receptor antagonists; PDE5i: phosphodiesterase -5 inhibitor; PAH: pulmonary arterial hypertension. \#: for continuous variables, hazard ratios reported corresponding to a $1 \mathrm{SD}$ increase in the variable.

\begin{tabular}{|c|c|c|c|}
\hline \multirow{2}{*}{$\begin{array}{l}\text { TABLE } 4 \\
\text { Covariate }\end{array}$} & \multicolumn{3}{|c|}{$\begin{array}{l}\text { Cardiac troponin I (cTnl) and risk of transplant or } \\
\text { right ventricular death when adjusted individually } \\
\text { for selected covariates }\end{array}$} \\
\hline & & cTnl adjusted HR $(95 \% \mathrm{Cl})$ & p-value \\
\hline \multicolumn{2}{|l|}{ Age yrs } & $4.36(1.72-11.02)$ & 0.002 \\
\hline \multicolumn{2}{|c|}{ IPAH versus APAH } & $4.06(1.57-10.53)$ & 0.004 \\
\hline \multicolumn{2}{|l|}{ NYHA class } & $3.84(1.48-9.96)$ & 0.006 \\
\hline \multicolumn{2}{|l|}{ 6MWD m } & $3.02(1.07-8.53)$ & 0.040 \\
\hline \multicolumn{2}{|c|}{ Pericardial effusion } & $3.81(1.49-9.75)$ & 0.005 \\
\hline \multicolumn{2}{|l|}{ Pra $\mathrm{mmHg}$} & $3.91(1.47-10.39)$ & 0.006 \\
\hline \multicolumn{2}{|c|}{$\mathrm{CI} \mathrm{L} \cdot \mathrm{min}^{-1} \cdot \mathrm{m}^{-2}$} & $4.75(1.89-11.91)$ & 0.001 \\
\hline \multicolumn{2}{|c|}{ Prostacyclin therapy } & $4.80(1.91-12.06)$ & 0.001 \\
\hline \multicolumn{2}{|l|}{ CRP $g \cdot L^{-1}$} & $3.80(1.47-9.84)$ & 0.006 \\
\hline \multicolumn{2}{|c|}{$\mathrm{HDL}-\mathrm{C} \mathbf{m g} \cdot \mathrm{dL}^{-1}$} & $2.88(1.04-7.97)$ & 0.042 \\
\hline \multicolumn{2}{|c|}{ Creatinine $\mathrm{mg} \cdot \mathrm{dL}^{-1}$} & $3.88(1.46-10.31)$ & 0.007 \\
\hline
\end{tabular}

HR: hazard ratio; IPAH: idiopathic pulmonary arterial hypertension; APAH: associated pulmonary arterial hypertension; NYHA: New York Heart Association functional class; 6MWD: 6-min walk distance; Pra: right atrial pressure; $\mathrm{Cl}$ : cardiac index; CRP: C-reactive protein; HDL-C: high-density lipoprotein cholesterol.

small numbers of patients in each group do not allow for firm conclusions. When analysing all-cause mortality and transplantation, detectable cTnI was still highly predictive of worse outcomes (HR 3.21, 95\% CI 1.40-7.37; p=0.004). When excluding the two patients with cTnI levels between 0.001 and $0.008 \mathrm{ng} \cdot \mathrm{mL}^{-1}$, detectable cTnI was still highly associated death by RVF (HR 4.07, 95\% CI 1.54-10.78; p <0.001) (see online supplementary figure 1 and table 1 ).

\section{DISCUSSION}

The main finding of our study is that cTnI detected by a sensitive assay even at subclinical levels is associated with more severe disease and worse transplant-free survival in patients with PAH. The current study uses a sensitive troponin assay and provides the largest cohort with a homogenous category $1 \mathrm{PAH}$ population treated with contemporary PAHtargeted therapies for the longest follow-up time.

The elevated pulmonary vascular resistance that characterises $\mathrm{PAH}$ leads to increase intramural right ventricular tension and eventually decreased cardiac output, which in turn can lead to lower systemic pressures. This situation is conducive to right ventricular myocardial ischaemia and necrosis, which may account for the troponin leak observed in a quarter of our patients. Our data support this notion, as we observed more right heart chamber dilation and dysfunction in patients with detectable cTnI. Mechanisms other than myocardial ischaemia are also possible. We found a positive correlation between CTnI and CRP levels, suggesting that inflammatory mechanisms may play a role. In the present study, a 1 SD increase in CRP levels was not associated with death by RVF (table 3). This contrasts with the report by QUARCK et al. [18] showing decreased survival with CRP levels above the upper limit of normal of $5 \mathrm{mg} \cdot \mathrm{L}^{-1}$. We have previously reported a similar association with regards to clinical worsening, but not survival [19]. While differences in the statistical methods used may 
account for these discrepancies, the relationship between CRP and clinical outcomes deserves further study.

The previous two studies of cardiac troponin in pulmonary hypertension are limited by a number of issues: the use of a lower-sensitivity assay [12]; the inclusion of heterogeneous populations with pulmonary hypertension secondary to chronic thromboembolic disease and interstitial lung disease $[12,13]$; low numbers of category 1 PAH subjects [13]; shortterm follow-up with small numbers of clinical events [13]; and noncontemporary management of PAH [20]. In the present study, we used an assay that provides highly sensitive analytical measurement of cTnI with a three-decimal point readout, which has been found to be comparable with other high-sensitivity assays [16]. We detected cTnI in $25 \%$ of the patients with the sensitive assay, and cTnT in $6.5 \%$ of patients, compared with a detection rate of $13-14 \%$ for cTnT in previous studies in Poland [12, 21], and $4.3 \%$ in a study of chronic thromboembolic pulmonary hypertension by LANKEIT et al. [22]. Our lower detection rates for cTnT may be explained by the inclusion of a majority of patients receiving PAH-targeted therapies. Our findings for CTnT mirror the results of LANKEIT et al. [22] (very low detection rate and poor outcomes in all patients with detectable cTnT) and suggest that using an assay with a two-decimal point readout for CTnT does not provide enough prognostic sensitivity. We have shown that the association between cardiac troponins and poor outcomes extends to lower troponin levels not detected with assays in current clinical use.

We report on a cohort that includes only diagnostic category 1 $\mathrm{PAH}$, according to the recent classification [14], who were treated with contemporary PAH management [20]. The latter may explain the 52.9\% 24-month survival rate in our cohort with detectable cTnI, compared with $29 \%$ in the report by TORBICKI et al. [12] published in 2003. Remarkably, even with improved outcomes, cTnI was predictive of transplant-free survival in our cohort. The sensitive assay used for this study, which allows for the detection of subclinical levels of cTnI, may have contributed to our ability to show this predictive ability. A recent French study failed to show any association between cTnI and survival in patients with acutely worsened $\mathrm{PAH}$, with the authors noting that more sensitive assays for troponin measurements should be used [23].

Detectable cTnI was associated with more severe PAH, as suggested by more advanced functional class symptoms, more pericardial effusions, shorter 6MWDs, higher Pra, larger RA area, higher BNP and CRP levels, and lower HDL-C concentrations. The detection of $\mathrm{CTnI}$ by our sensitive assay carried a 4.7-fold increase risk of death related to RVF or transplant, with 36-month transplant-free survival of $44.1 \%$, compared with $84.7 \%$ in patients with undetectable cTnI. cTnI was predictive of worse outcomes when adjusted individually for several covariates known to influence survival in $\mathrm{PAH}$, including age, idiopathic versus associated $\mathrm{PAH}$, functional class, 6MWD, pericardial effusion, Pra, cardiac index, creatinine, CRP and HDL-C. As they both reflect right ventricular strain, it is not surprising that we found a strong correlation between cTnI and BNP. Furthermore, we found a significant interaction between these markers (data not shown). The addition of cTnI to BNP may allow for better risk stratification.
The study of a relatively small and heterogeneous PAH population, including idiopathic disease and cases associated with connective tissue disease, constitutes a limitation of our study, and one that is common to single-centre biomarker investigations. Nevertheless, when adjusted for PAH category (idiopathic versus associated disease), cTnI remained associated with worse outcomes. Another limitation of this study is the inclusion of incident and prevalent cases, where $75 \%$ of the patients were receiving PAH-targeted therapies when cardiac troponins were measured. While this may have biased the results and also explains the very low detection rate for cTnT, it is noteworthy that a more sensitive troponin assay still provided prognostic information in patients receiving contemporary PAH therapies. Before a novel biomarker can be recommended for widespread clinical use, it needs to be evaluated in a rigorous fashion [24]. While biological plausibility certainly exists for the use of circulating troponins in $\mathrm{PAH}$, and the available evidence suggests a potential role, further studies with larger numbers of patients are needed to confirm their utility. For example, a recent systematic review of the literature suggested that troponins may not be discriminative enough to be used in the risk stratification of patients with normotensive acute pulmonary embolism [25]. As cardiac troponin assays become more sensitive, cut-offs associated with meaningful clinical outcomes will have to be defined to avoid diagnostic confusion. For instance, in patients suspected of having acute myocardial infarction, the higher-sensitivity assays improve diagnostic sensitivity, but at the expense of lower specificity $[16,26]$. Recently, cTnT has been detected in $25 \%$ of the general population [27] and $66 \%$ in people aged $>65$ yrs [28] using highly sensitive assays with a limit of detection of $0.003 \mathrm{ng} \cdot \mathrm{mL}^{-1}$. Rates of detection are much lower in females and young people [27], which is relevant to patients afflicted with PAH.

In summary, using a sensitive assay, we found a $25 \%$ detection rate for $\mathrm{CTnI}$ in a cohort of category $1 \mathrm{PAH}$ patients. Detectable cTnI was associated with more severe PAH and worse clinical outcomes. The measurement of circulating cTnI with a sensitive assay may help clinicians to identify high-risk patients who could benefit from more aggressive therapies.

\section{STATEMENT OF INTEREST}

Statements of interest for G.A. Heresi, W.H.W. Tang, S.L. Hazen and R.A. Dweik can be found at www.erj.ersjournals.com $/ \mathrm{site} / \mathrm{misc} /$ statements.xhtml

\section{REFERENCES}

1 Bogaard HJ, Abe K, Vonk Noordegraaf A, et al. The right ventricle under pressure: cellular and molecular mechanisms of right-heart failure in pulmonary hypertension. Chest 2009; 135: 794-804.

2 Humbert M, Sitbon O, Yaici A, et al. Survival in incident and prevalent cohorts of patients with pulmonary arterial hypertension. Eur Respir J 2010; 36: 549-555.

3 Badesch DB, Abman SH, Simonneau G, et al. Medical therapy for pulmonary arterial hypertension: updated ACCP evidence-based clinical practice guidelines. Chest 2007; 131: 1917-1928.

4 Warwick G, Thomas PS, Yates DH. Biomarkers in pulmonary hypertension. Eur Respir J 2008; 32: 503-512.

5 Morrow DA, Cannon CP, Jesse RL, et al. National Academy of Clinical Biochemistry Laboratory Medicine Practice Guidelines: 
clinical characteristics and utilization of biochemical markers in acute coronary syndromes. Circulation 2007; 115: e356-e375.

6 Eggers KM, Lagerqvist B, Venge $\mathrm{P}$, et al. Persistent cardiac troponin I elevation in stabilized patients after an episode of acute coronary syndrome predicts long-term mortality. Circulation 2007; 116: 1907-1914.

7 Horwich TB, Patel J, MacLellan WR, et al. Cardiac troponin I is associated with impaired hemodynamics, progressive left ventricular dysfunction, and increased mortality rates in advanced heart failure. Circulation 2003; 108: 833-838.

8 Apple FS, Murakami MM, Pearce LA, et al. Predictive value of cardiac troponin I and $\mathrm{T}$ for subsequent death in end-stage renal disease. Circulation 2002; 106: 2941-2945.

9 Ammann P, Fehr T, Minder EI, et al. Elevation of troponin I in sepsis and septic shock. Intensive Care Med 2001; 27: 965-969.

10 Quenot JP, Le Teuff G, Quantin C, et al. Myocardial injury in critically ill patients: relation to increased cardiac troponin I and hospital mortality. Chest 2005; 128: 2758-2764.

11 Janata KM, Leitner JM, Holzer-Richling N, et al. Troponin $\mathrm{T}$ predicts in-hospital and 1-year mortality in patients with pulmonary embolism. Eur Respir J 2009; 34: 1357-1363.

12 Torbicki A, Kurzyna M, Kuca P, et al. Detectable serum cardiac troponin $\mathrm{T}$ as a marker of poor prognosis among patients with chronic precapillary pulmonary hypertension. Circulation 2003; 108: 844-848.

13 Filusch A, Giannitsis E, Katus HA, et al. High-sensitive troponin T: a novel biomarker for prognosis and disease severity in patients with pulmonary arterial hypertension. Clin Sci (Lond) 2010; 119: 207-213.

14 Simonneau G, Robbins IM, Beghetti M, et al. Updated clinical classification of pulmonary hypertension. J Am Coll Cardiol 2009; 54: Suppl., S43-S54.

15 Tang WH, Wu Y, Nicholls SJ, et al. Subclinical myocardial necrosis and cardiovascular risk in stable patients undergoing elective cardiac evaluation. Arterioscler Thromb Vasc Biol 2010; 30: 634-640.

16 Reichlin T, Hochholzer W, Bassetti S, et al. Early diagnosis of myocardial infarction with sensitive cardiac troponin assays. N Engl J Med 2009; 361: 858-867.

$17 \mathrm{R}$ Development Core Team. R: A language and environment for statistical computing. R Foundation for Statistical Computing V,
Austria, 2008. www.R-project.org Date last accessed: November 21, 2011.

18 Quarck R, Nawrot T, Meyns B, et al. C-reactive protein: a new predictor of adverse outcome in pulmonary arterial hypertension. J Am Coll Cardiol 2009; 53: 1211-1218.

19 Heresi GA, Aytekin M, Newman J, et al. Plasma levels of highdensity lipoprotein cholesterol and outcomes in pulmonary arterial hypertension. Am J Respir Crit Care Med 2010; 182: 661-668.

20 Galie N, Hoeper MM, Humbert M, et al. Guidelines for the diagnosis and treatment of pulmonary hypertension: The Task Force for the Diagnosis and Treatment of Pulmonary Hypertension of the European Society of Cardiology (ESC) and the European Respiratory Society (ERS), endorsed by the International Society of Heart and Lung Transplantation (ISHLT). Eur Heart J 2009; 30: 2493-2537.

21 Fijalkowska A, Kurzyna M, Torbicki A, et al. Serum N-terminal brain natriuretic peptide as a prognostic parameter in patients with pulmonary hypertension. Chest 2006; 129: 1313-1321.

22 Lankeit M, Dellas C, Panzenbock A, et al. Heart-type fatty acidbinding protein for risk assessment of chronic thromboembolic pulmonary hypertension. Eur Respir J 2008; 31: 1024-1029.

23 Sztrymf B, Souza R, Bertoletti L, et al. Prognostic factors of acute heart failure in patients with pulmonary arterial hypertension. Eur Respir J 2010; 35: 1286-1293.

24 Heresi GA. Clinical perspective: biomarkers in pulmonary arterial hypertension. Int J Clin Pract Suppl 2011; 169: 5-7.

25 Jimenez D, Uresandi F, Otero R, et al. Troponin-based risk stratification of patients with acute nonmassive pulmonary embolism: systematic review and metaanalysis. Chest 2009; 136: 974-982.

26 Keller T, Zeller T, Peetz D, et al. Sensitive troponin I assay in early diagnosis of acute myocardial infarction. $N$ Engl J Med 2009; 361: 868-877.

27 de Lemos JA, Drazner MH, Omland T, et al. Association of troponin $\mathrm{T}$ detected with a highly sensitive assay and cardiac structure and mortality risk in the general population. JAMA 2010; 304: 2503-2512.

28 deFilippi CR, de Lemos JA, Christenson RH, et al. Association of serial measures of cardiac troponin $\mathrm{T}$ using a sensitive assay with incident heart failure and cardiovascular mortality in older adults. JAMA 2010; 304: 2494-2502. 